\title{
A naive toy model of dark matter: Fermion sea
}

\author{
ZHANG YuJie \\ Key Laboratory of Micro-nano Measurement-Manipulation and Physics (Ministry of Education) and School of Physics, Beihang University, \\ Beijing 100191, China (email: nophy0@gmail.com)
}

Received October 26, 2010; accepted December 14, 2010; published online April 7, 2011

\begin{abstract}
A naive toy model that the fermion sea offers the dark matter density is discussed here. If the fermion sea fills the total space and the temperature equals the temperature of $\mathrm{CMB}$, we can get the mass of the fermion is about $4.7 \times 10^{-38} \mathrm{~kg}(0.026 \mathrm{eV})$.
\end{abstract}

dark matter, fermion sea, CMB temperature

PACS: 98.80.Bp, 95.35.+d

The dark matter density and the CMB temperature are [1]

$$
\begin{aligned}
& \rho_{\text {DM }} \sim 0.22 \times 10^{-29} \mathrm{~g} / \mathrm{cm}^{3}, \\
& T_{\mathrm{CMB}}=2.725 \pm 0.001 \mathrm{~K} .
\end{aligned}
$$

We assume the temperature of thermal motion of the fermion is the temperature of CMB,

$$
k T_{\mathrm{CMB}}=\frac{m c^{2}}{\sqrt{1-v^{2} / c^{2}}}-m c^{2} .
$$

Here $m$ is the fermion mass, and $v$ is the velocity. The corresponded de Broglie wave is

$$
\begin{gathered}
h / \lambda=\frac{m v}{\sqrt{1-v^{2} / c^{2}}}, \\
\lambda=\frac{h}{\sqrt{2 m k T_{\mathrm{CMB}}} \sqrt{1+k T_{\mathrm{CMB}} /\left(2 m c^{2}\right)}} .
\end{gathered}
$$

We also assume all the fermions stay at the ground state. The excited states should translate into the ground state in a long time. There are only two fermions in a cube with one $\lambda$ on each side, for the spin of fermion is $1 / 2$. We can get the density of the fermion sea

$$
\rho_{\mathrm{FS}}=\frac{2 m}{\lambda^{3}}=\frac{(2 m)^{5 / 2}\left(k T_{\mathrm{CMB}}\right)^{3 / 2}}{h^{3} \sqrt{1-v^{2} / c^{2}}}\left(1+\frac{k T_{\mathrm{CMB}}}{2 m c^{2}}\right)^{3 / 2} .
$$

The fermion sea fills the total space. If we consider the fermion sea offers the dark matter density

$$
\rho_{\mathrm{FS}}=\rho_{\mathrm{DM}},
$$

we can get

$$
v=4.0 \times 10^{7} \mathrm{~m} / \mathrm{s}=0.13 c,
$$

$$
\begin{aligned}
& m=4.7 \times 10^{-38} \mathrm{~kg}=0.026 \mathrm{eV} / c^{2}, \\
& w=p / \rho \approx v^{2} / c^{2} / 3=0.0058 .
\end{aligned}
$$

For $w \sim 0$, it should be cold dark matter. Within the Standard Model, the mass of neutrino may be compared with this value. But the cross section between the neutrino and the photon is very small and the neutrino has been decoupled at the early universe. Of course, this is a naive, semiclassical toy model. The effect of Quantum and General Relativity should be taken into account. And many related questions should be studied: Where are bosons and other fermions? What is the fermion? Why the temperature of CMB is $2.7 \mathrm{~K}$ ? Why the mass of the fermion is $0.026 \mathrm{eV} / \mathrm{c}^{2}$ ? And so on.

In summary, we discussed a naive toy model that fermion sea offers the dark matter density. We assumed that the temperature of thermal motion of fermion is the temperature of $\mathrm{CMB}$, and only two fermions in a cube one wavelength of de Broglie wave on each side. If we select the mass of fermion $4.7 \times 10^{-38} \mathrm{~kg}\left(0.026 \mathrm{eV} / \mathrm{c}^{2}\right)$, the density of the fermion sea equals the dark matter density.

This work was supported by the National Natural Science Foundation of China (Grant Nos. 10805002 and 11075011), the Foundation for the Author of National Excellent Doctoral Dissertation of China (Grant No. 201020), and the Fundamental Research Funds for the Central Universities.

1 Amsler C, Doser M, Antonelli M, et al. (Particle Data Group) Review of particle physics. Phys Lett B, 2008, 667: 1-1340 